\title{
Primary Fusiform Superior Vena Cava Aneurysm
}

\author{
Rajesh Sharma ${ }^{a}$,, Manoj Ravi ${ }^{\mathrm{a}}$, T. Govindan Unni ${ }^{\mathrm{a}}$
}

\begin{abstract}
Superior vena cava (SVC) aneurysms are rare mediastinal vascular lesions. We report a case of a 42-year-old female, who presented to the outpatient department with features suggestive of lower respiratory tract infection. Chest X-ray showed abnormal contour and widening of right border of mediastinum. Computed tomography (CT) thorax revealed fusiform aneurysmal ectasia of SVC measuring $4.5 \times$ $5.5 \times 8.9 \mathrm{~cm}$ without internal thrombosis or dissecting flap. Management options include observation with follow-up and in some cases anticoagulation and surgical excision may be considered. The general consensus is that fusiform variety can be managed conservatively in view of the low risk of complications. The saccular aneurysms may need to be managed with anticoagulation therapy or surgically in view of the possible risk for thrombus formation and pulmonary embolism. Since in our case it was an asymptomatic primary fusiform SVC aneurysm, patient was advised for conservative management and follow-up.
\end{abstract}

Keywords: Superior vena cava; Aneurysm; Fusiform

\section{Introduction}

Aneurysms of superior vena cava (SVC) are very rare mediastinal vascular lesions. The diagnosis of this lesion is often missed because of its rarity. Only 37 cases are reported worldwide till date in available literature [1]. The first case was reported by Abbot in 1950, a fusiform aneurysm of SVC [2]. Two categories of SVC aneurysms are described, fusiform and saccular aneurysms. SVC aneurysms are generally asymptomatic and are often incidental finding on imaging studies. They generally are not associated with specific symptoms. Complications are very rare overall, with theoretical possibility of thrombus formation and pulmonary embolism with saccular aneurysm. Management options include mostly observation with follow-up, while in some cases anticoagulation and

Manuscript submitted July 1, 2017, accepted July 25, 2017

aDepartment of Cardiology, Jubilee Mission Medical College \& Research Institute, Thrissur, Kerala, India

${ }^{b}$ Corresponding Author: Rajesh Sharma, Department of Cardiology, Jubilee Mission Medical College \& Research Institute, Thrissur 680005, Kerala, India. Email: drrajeshsharma8@gmail.com

doi: https://doi.org/10.14740/cr570w surgical excision may be considered.

\section{Case Report}

A 42-year-old female was referred to the outpatient department, with features suggestive of lower respiratory tract infection. Physical examination was unremarkable. She had no history of any cardiac disease or injury to thoracic cage. She had no history of antecedent serious illness. Family history was also non-contributory.

Chest X-ray was done for the workup of respiratory symptoms, which showed abnormal contour and widening of right border of mediastinum (Fig. 1). In chest X-ray, the cervicothoracic sign and hilum overlay sign were present in posteroanterior (PA) view. Lateral view showed anterior bulging of mediastinum.

Computed tomography (CT) of thorax was done for further workup in angiographic protocol which was suggestive of aneurysmal ectasia of SVC and patchy consolidation of right lower lobe. Aneurysmal ectasia of SVC measuring $4.5 \times 5.5$ $\mathrm{cm}$ in maximum dilatation and for a length of $8.9 \mathrm{~cm}$ without internal thrombosis or dissecting flap was present (Figs. 2 and

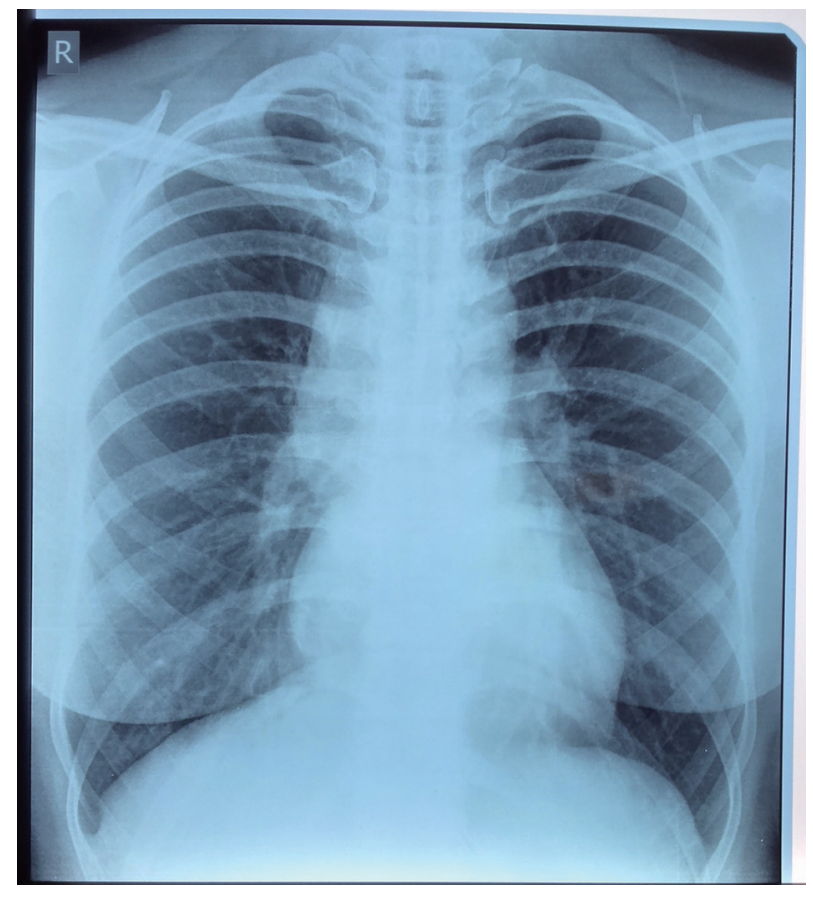

Figure 1. Chest $\mathrm{X}$-ray (PA) view showing right mediastinal widening. 


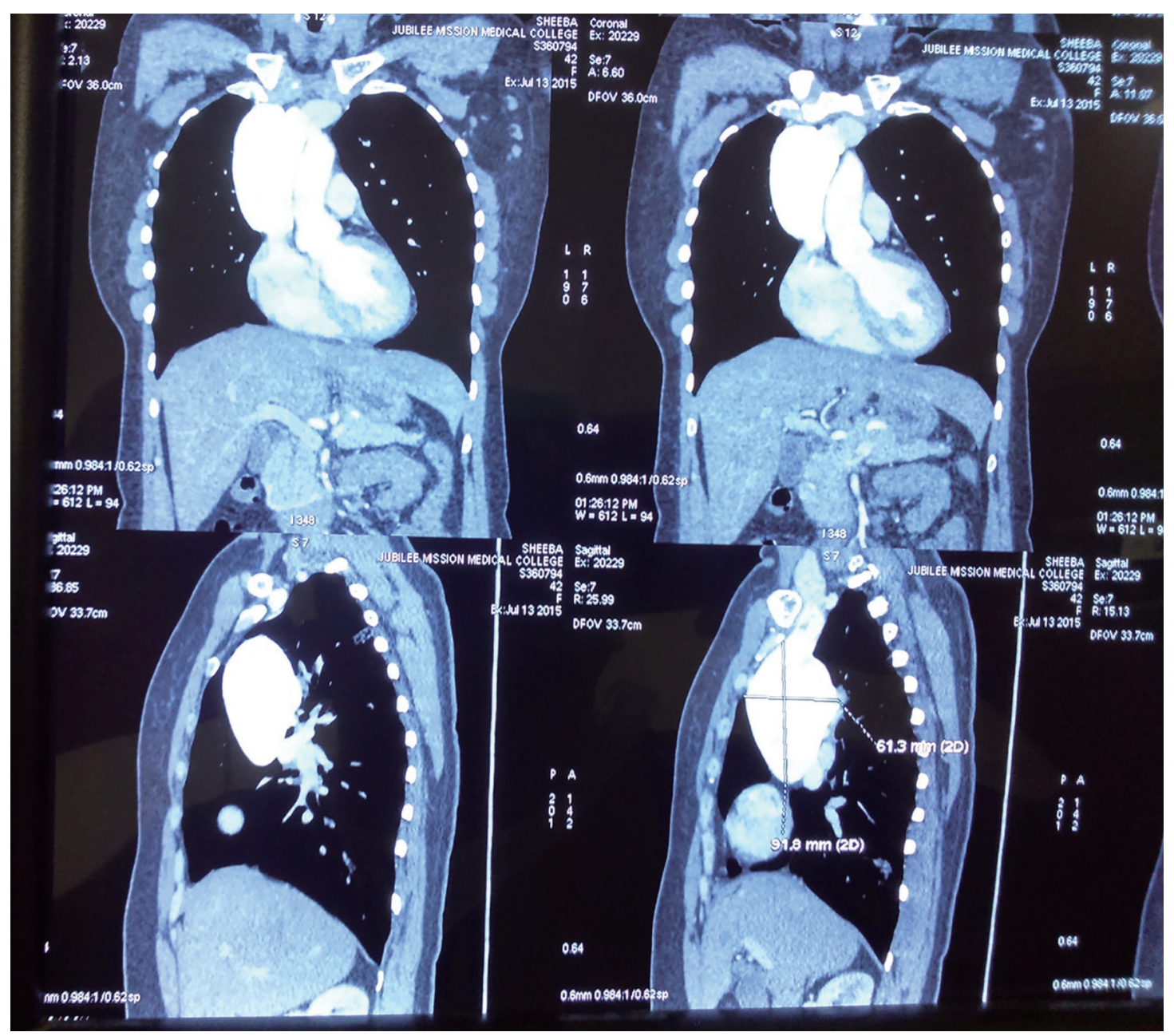

Figure 2. CT (thorax) of coronal and sagittal section showing SVC aneurysm.

3). No other vascular anomalies were detected.

Since CT did not reveal any signs of obstruction, we believe that the SVC aneurysm is primary and can be classified as congenital primary superior vena caval aneurysm (CPSVCA), class 1A as per Abott's classification (1964) [3].

\section{Discussion}

SVC aneurysms are one of the rare phenomena with approximately 37 cases reported till date [1]. To date, SVC aneurysms as a group have an equal sex incidence. It lacks a definable predisposing cause, resulting in diagnostic and therapeutic problem. Aneurysms may be congenital, or caused by inflammation or by the degeneration of the vascular wall, but in most cases, their cause is unknown [4]. SVC aneurysms are generally asymptomatic and are often incidental finding on imaging studies [5]. In earlier case reports, exploratory thoracotmy and venogram were used for diagnosis. In present era, tests that may lead to the correct diagnosis include roentgenograms, CT, chest fluoroscopy, and nuclear flow scans [6].

The conventional radiographic findings may also suggest a venous nature of a mediastinal mass. A positional variation of size of the mass in chest radiography is reported in venous aneurysms of mediastinum, but this test is not often done because of failure to appreciate this possibility [5]. Reports indicated enlargement on expiration, on Valsalva maneuver, or in recumbent position in fusiform aneurysms of the SVC [7]. The cervico-thoracic sign (disappearance of mass as it approaches the clavicle) and the hilum overlay sign (visibility of the right hilum through the mass) suggest that the mass is mainly anteriorly located in mediastinum. CT scan usually demonstrates a contrast enhancing lesion adjacent to the ascending aorta. The enhancement approaches but is less than that of aorta. Sometimes, the lesion shows calcification and may be mistaken for part of thymic gland $[5,6]$.

Treatment of SVC aneurysm is based on type and size of the venous ectasia. Most of the authors prefer conservative management with follow-up. Generally, asymptomatic fusiform aneurysms without thrombus are just monitored over time. Developing a treatment plan for an individual with a saccular aneurysm requires more consideration. Use of longterm anticoagulation or antiplatelets to prevent thrombus and, therefore, prevent pulmonary thromboembolism is debatable, 


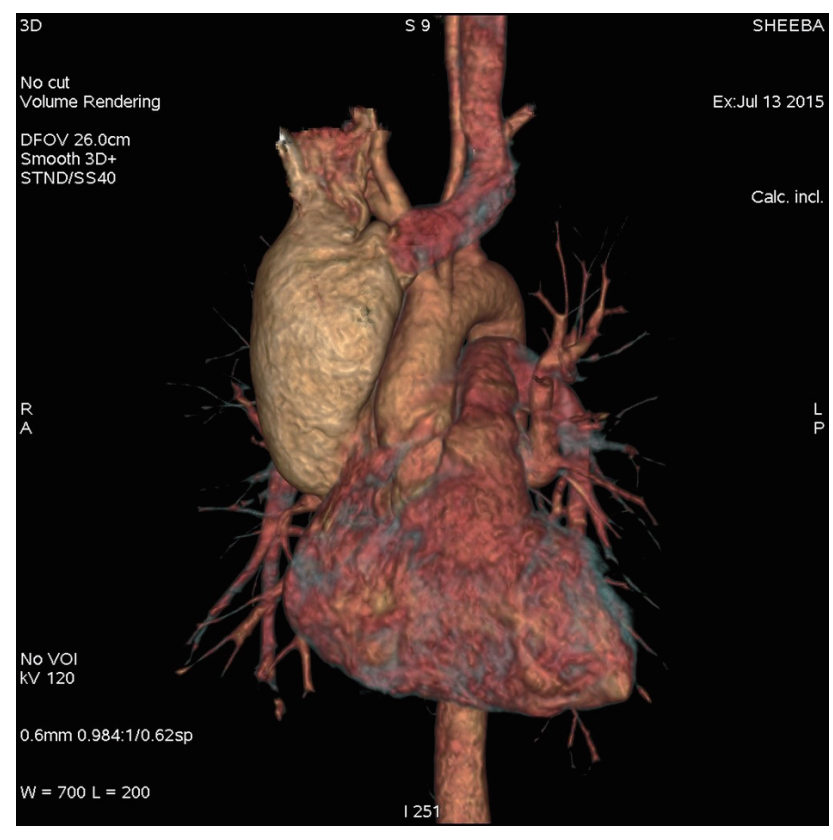

Figure 3. 3D reconstructed image of CT (thorax) in angiographic protocol demonstrating SVC aneurysm.

because as the aneurysm grows in size, the risk of rupture increases. Anticoagulation with the above agents in that setting of aneurysm rupture increases morbidity and mortality. In cases of larger saccular SVC aneurysms, even those that are asymptomatic, prophylactic surgical resection has been recommended [1].

The long-term prognosis seems to be good even without surgery, hence many authors argue for conservative management in these lesions. In SVC aneurysms, only three complications have been reported: two episodes of pulmonary embolism and one that developed contained rupture $[6,8,9]$. Most authors are of the opinion that CPSVCA should be managed conservatively, because they do not enlarge or produce pressure symptoms or rupture, and spontaneous thromboembolism is exceptional. It is postulated that they do not produce pressure symptoms or rupture spontaneously because of the low systemic venous pressure. A low incidence of spontaneous thromboembolism is to be expected, since the blood flow inside the aneurysm, fusiform or saccular, is not sluggish as demonstrated by the normal transit time on radioisotopic angiocardiography [6].

In conclusion, since in our case it was an asymptomatic primary fusiform SVC aneurysm, patient was advised for con- servative management and follow-up.

\section{Acknowledgments}

We extend our sincere gratitude to faculty members of Jubilee Center for Medical Research for editing this article.

\section{Grant Support}

None.

\section{Conflicts of Interest}

None.

\section{References}

1. Patel A, Cobb R, Rivera V, Simpson S. Untreated superior vena cava aneurysm: radiological significance and review of the literature. Case Rep Radiol. 2016;2016:6960757.

2. Kelley MJ, Mannes EJ, Ravin CE. Mediastinal masses of vascular origin. A review. J Thorac Cardiovasc Surg. 1978;76(4):559-572.

3. Abbott OA. Congenital aneurysm of superior vena cava; report of one case with operative correction. Ann Surg. 1950;131(2):259-263.

4. Pasic M, Schopke W, Vogt P, von Segesser L, Schneider $\mathrm{J}$, Turina M. Aneurysm of the superior mediastinal veins. J Vasc Surg. 1995;21(3):505-509.

5. Varma PK, Dharan BS, Ramachandran P, Neelakandhan KS. Superior vena caval aneurysm. Interact Cardiovasc Thorac Surg. 2003;2(3):331-333.

6. Modry DL, Hidvegi RS, LaFleche LR. Congenital saccular aneurysm of the superior vena cava. Ann Thorac Surg. 1980;29(3):258-262.

7. Hidvegi RS, Modry DL, LaFleche L. Congenital saccular aneurysm of the superior vena cava: radiolographic features. AJR Am J Roentgenol. 1979;133(5):924-927.

8. Gaucher L, Dezile G, Roullier A, Bouvier B, Aloin M, Delvert JC, Belda G, et al. [Saccular aneurysm of the superior vena cava]. Rev Mal Respir. 1988;5(6):637-639.

9. Yokomise H, Nakayama S, Aota M, Daitoh N, Katsura H. Systemic venous aneurysms. Ann Thorac Surg. 1990;50(3):460-462. 